\title{
DESEMPENHO TÉRMICO DE SISTEMAS INTEGRADOS DE BIO-OXICOMBUSTÃO COM A INJEÇÃO DE DIFERENTES COMBURENTES
}

\author{
I. A. SEVERO ${ }^{1}$, N. H. NEUENFELDT ${ }^{1}$, M. C. DEPRÁ ${ }^{1}$, L. Q. ZEPKA e E. JACOB-LOPES ${ }^{1}$ \\ ${ }^{1}$ Universidade Federal de Santa Maria, Departamento de Tecnologia e Ciência de Alimentos \\ E-mail para contato: jacoblopes@pq.cnpq.br
}

\begin{abstract}
RESUMO - O objetivo do trabalho foi avaliar o desempenho térmico do sistema integrado de bio-oxicombustão com a injeção de diferentes comburentes. Os experimentos foram realizados em um fotobiorreator de coluna de bolhas com volume total de trabalho de $2 \mathrm{~L}$, intensidade luminosa de $150 \mu \mathrm{mol} \cdot \mathrm{m}^{-2} \mathrm{~s}^{-1}$ e injeção de ar enriquecido com $15 \%$ de dióxido de carbono. A microalga utilizada foi a Scenedesmus obliquus e as condições de cultivo foram: meio BG11, fotoperíodo de $12 \mathrm{~h}$, concentração inicial de células de $100 \mathrm{mg} / \mathrm{L}$, temperatura de $25^{\circ} \mathrm{C}$, aeração contínua de $1 \mathrm{VVM}$ e pH inicial de 7,6. Um forno de oxicombustão foi projetado em escala laboratorial. O combustível utilizado foi o coque de petróleo. Uma corrente gasosa industrial simulada contendo $5,5 \% \mathrm{O}_{2}, 18,0 \% \mathrm{CO}_{2}$ e $76,5 \%$ $\mathrm{N}_{2}$, ar atmosférico e os gases de exaustão do fotobiorreator foram utilizados como comburentes. As condições experimentais foram: massa de coque de 1,0 g, tempo de combustão de 20 min e taxa de aeração de $1,0 \mathrm{~L} / \mathrm{min}$. O monitoramento da temperatura foi feito por termografia a cada $24 \mathrm{~h}$ durante a fase de crescimento do micro-organismo. Os resultados demonstraram que através da injeção dos gases de exaustão do fotobiorreator obteve-se um aumento na temperatura de $30,5 \%$ e $45,8 \%$ superiores ao uso do ar atmosférico e da corrente gasosa industrial simulada, respectivamente. Nesse sentido, a integração de processos demonstrou ser uma potencial abordagem de engenharia para promover a sustentabilidade dos processos industriais.
\end{abstract}

\section{INTRODUÇÃO}

As emissões antropogênicas de poluentes atmosféricos pela queima de combustíveis fósseis são responsáveis pela mudança climática global. $\mathrm{O}$ dióxido de carbono $\left(\mathrm{CO}_{2}\right)$, por exemplo, tem impacto negativo representando a maior parcela destas emissões (IPCC, 2014). Diante disso, uma forma de mitigar esse problema é através do desenvolvimento de tecnologias para a captura, armazenamento ou utilização de carbono visando à estabilização das emissões de $\mathrm{CO}_{2}$ (Cuéllar-Franca e Azapagic, 2015).

A oxicombustão é considerada uma rota tecnológica promissora para este propósito, a qual consiste em substituir o ar utilizado nos processos convencionais de combustão por atmosferas enriquecidas de oxigênio. Como resultado, há um ganho na capacidade térmica, resultando em uma elevada eficiência energética dos equipamentos (Yin e Yan, 2016). 
Entretanto, a obtenção de oxigênio de alta pureza é cara e apresenta um alto consumo energético (Jin et al., 2015).

Para contornar esse problema, a aplicação de fotobiorreatores (FBRs) microalgais pode ser uma opção viável. As microalgas possuem como co-produto metabólico o oxigênio gerado através das reações de fotólise da água. Paralelamente, diversos compostos orgânicos voláteis (COVs) são produzidos, apresentando considerável valor energético, além de liberarem nos gases de exaustão substanciais concentrações de $\mathrm{CO}_{2}$ (Jacob-Lopes et al., 2010).

Por tanto, a integração de processos é necessária, tanto para fornecer o oxigênio requerido nos processos de oxicombustão, quanto para capturar $\mathrm{CO}_{2}$, através da biooxicombustão. Essa técnica consiste em gerar biologicamente oxigênio, $\mathrm{COVs}$ e $\mathrm{CO}_{2}$, os quais são reutilizados como comburente, combustíveis gasosos e diluentes de nitrogênio, respectivamente, em equipamentos industrias de combustão, para maximizar a eficiência energética desses sistemas (Jacob-Lopes et al., 2015). Nesse sentido, o objetivo do trabalho foi avaliar o desempenho térmico do sistema integrado de bio-oxicombustão com a injeção de diferentes comburentes.

\section{MATERIAL E MÉTODOS}

\subsection{Micro-organismo e meio de cultura}

A espécie utilizada foi a Scenedesmus obliquus (CPCC5) obtida da Canadian Phycological Culture Centre. As culturas estoque foram propagadas e mantidas em meio sintético BG11 (Rippka et al., 1979) e pH 7,6. As condições de incubação usadas foram: temperatura de $25^{\circ} \mathrm{C}$, densidade de fluxo de fótons de $15 \mu \mathrm{mol} / \mathrm{m}^{2} / \mathrm{s}$, fotoperíodo de $12 \mathrm{~h}$ e agitação constante de 1 VVM (volume de ar por volume de cultura por minuto).

\subsection{Fotobiorreator e condições de cultivo}

Os experimentos foram realizados em um fotobiorreator de coluna de bolhas (Maroneze et al., 2016), operando em regime intermitente, alimentados com $2 \mathrm{~L}$ de meio de cultura. As condições experimentais foram: concentração celular inicial de $100 \mathrm{mg} / \mathrm{L}$, temperatura de $25^{\circ} \mathrm{C}$, densidade de fluxo de fótons de $150 \mu \mathrm{mol} / \mathrm{m}^{2} / \mathrm{s}$ e aeração contínua de 1VVM com injeção de ar enriquecido com $15 \%$ de dióxido de carbono. A concentração celular foi monitorada a cada $24 \mathrm{~h}$ durante a fase de crescimento do micro-organismo. Os testes foram realizados em triplicata.

\subsection{Métodos analíticos}

A temperatura e o $\mathrm{pH}$ do meio de cultura foram determinadas através de um sensor polarográfico (Mettler Toledo, Suíça). A concentração celular foi determinada gravimetricamente, através da filtração de um volume conhecido de meio de cultura em filtro de diâmetro $0,45 \mathrm{~mm}$, seco a $60^{\circ} \mathrm{C}$ por $24 \mathrm{~h}$ (APHA, 2005). A intensidade luminosa foi determinada por um sensor quântico (Apogee, Logan-UT, EUA). 


\subsection{Sistema de bio-oxicombustão}

Os experimentos foram realizados em um sistema de bio-oxicombustão construído em escala laboratorial como é mostrado na Figura 1. O aparato experimental é constituído de um forno de aço inox, sendo seu revestimento composto por material refratário. A câmara possui $3 \mathrm{~cm}$ de espessura na parte interna, uma altura de $40 \mathrm{~cm}$, comprimento de $20 \mathrm{~cm}$ e largura de $20 \mathrm{~cm}$. O forno foi equipado com duas resistências elétricas de $300 \mathrm{~W}$. Um suporte cerâmico foi inserido no centro da câmara de combustão que foi utilizada para a introdução da amostra. Os gases de exaustão do fotobiorreator foram encaminhados ao forno através de uma tubulação de aço inox, localizado na parte inferior, com $80 \mathrm{~cm}$ de comprimento e $3 \mathrm{~mm}$ de diâmetro interno. Os gases resultantes da combustão foram encaminhados pelo canal de saída, localizado na parte superior da câmara. O sistema apresenta um filtro para umidade e duas bombas para controle da vazão de gases.

Figura 1 - Diagrama esquemático do sistema de bio-oxicombustão.

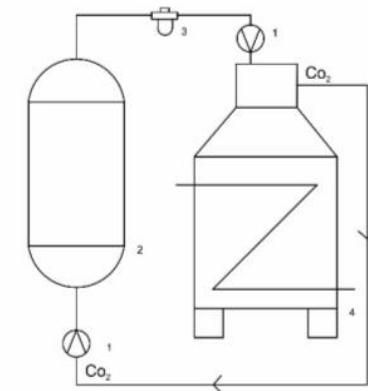

(A)

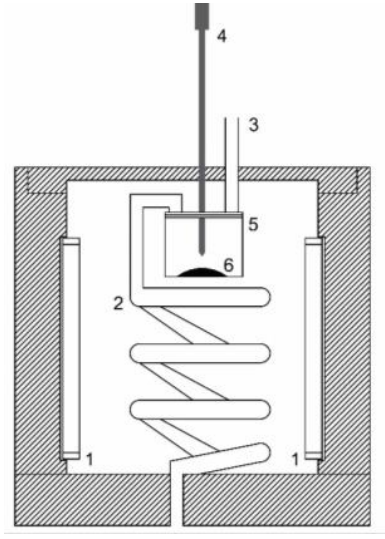

(B)

(A) 1: Bombas para controle da vazão e mistura dos gases; 2: Fotobiorreator; 3: Filtro para umidade; 4: Forno de bio-oxicombustão; (B) 1: Resistências elétricas; 2: Canal de entrada de $\mathrm{O}_{2}$; 3: Canal de saída dos gases de exaustão; 4: Sensor térmico; 5: Suporte cerâmico; 6: Câmara de combustão.

\subsection{Combustível e comburentes}

O combustível utilizado foi o coque de petróleo. A amostra foi caracterizada através da análise elementar usando um analisador elementar Perkin Elmer 2400 CHNS/O (Perkin Elmer, Waltham-MA, EUA), onde $2 \mathrm{mg}$ de coque foram oxidadas a $1000^{\circ} \mathrm{C}$ e os gases resultantes foram determinados por condutividade térmica. Acetanilida foi usada como padrão de referência contendo $71,09 \%$ carbono, $11,84 \%$ oxigênio, $6,71 \%$ hidrogênio e $10,36 \%$ nitrogênio. Os comburentes utilizados foram: uma corrente gasosa industrial simulada contendo $5,5 \% \mathrm{O}_{2}, 18,0 \% \mathrm{CO}_{2}$ e $76,5 \% \mathrm{~N}_{2}$, o ar atmosférico e os gases de exaustão do fotobiorreator.

\subsection{Obtenção das informações cinéticas no forno de bio-oxicombustão}


Os experimentos foram monitorados a cada $24 \mathrm{~h}$ durante a fase de crescimento do micro-organismo, através da injeção dos diferentes comburentes no forno de biooxicombustão e em diferentes tempos de residência celular. As condições experimentais foram: massa inicial de coque de 1,0 g, tempo de combustão de 20 min e taxa de aeração de $1,0 \mathrm{~L} / \mathrm{min}$. Durante a oxicombustão, a temperatura de chama foi continuamente monitorada. Cada ensaio foi realizado em triplicata.

\subsection{Desempenho térmico do sistema de bio-oxicombustão}

O monitoramento da temperatura de chama foi realizado através da imagem térmica da reação de combustão utilizando uma câmera no infravermelho Flir SC 305 (Flir Systems, Wilsonville-OR, EUA), obtendo imagens de $320 \times 240$ pixels a uma taxa de $3,75 \mathrm{~Hz}(3,75$ imagens térmicas por segundo). O alinhamento da câmera com a zona de combustão foi a uma distância de $60 \mathrm{~cm}$. As imagens foram processadas utilizando o software Flir Toolst.

\subsection{Análise Estatística}

A análise de variância (ANOVA) e o teste de Tukey $(\rho<0,05)$ foram utilizadas para testar as diferenças entre os comburentes estudados. As análises foram realizadas com o software Statistic 7.0 (StatSoft, Tulsa-OK, EUA).

\section{RESULTADOS E DISCUSSÃO}

Na Tabela 1 é mostrada a análise da dinâmica da temperatura de chama no sistema de bio-oxicombustão com a injeção de diferentes comburentes e em diferentes tempos de residência celular.

Tabela 1 - Dinâmica da temperatura de chama no sistema de bio-oxicombustão

\begin{tabular}{lcccccc}
\hline Parâmetro & $\begin{array}{c}5,5 \% \mathrm{O}_{2} ; 17 \% \\
\mathrm{CO}_{2} ; 76,5 \% \mathrm{~N}_{2}\end{array}$ & $\begin{array}{c}\mathrm{Ar} \\
\text { atmosférico }\end{array}$ & $\begin{array}{c}\text { FBR } \\
(0 \mathrm{~h})\end{array}$ & $\begin{array}{c}\text { FBR } \\
(48 \mathrm{~h})\end{array}$ & $\begin{array}{c}\text { FBR } \\
(96 \mathrm{~h})\end{array}$ & $\begin{array}{c}\text { FBR } \\
(144 \mathrm{~h})\end{array}$ \\
\hline $\begin{array}{l}\text { Temperatura } \\
\text { de chama }\left({ }^{\circ} \mathrm{C}\right)\end{array}$ & $822,8^{\mathrm{f}} \pm 8,2$ & $907,3^{\mathrm{d}} \pm 7,0$ & $925,9^{\mathrm{c}} \pm 16,6$ & $929,9^{\mathrm{b}} \pm 13,8$ & $1003,5^{\mathrm{a}} \pm 62,4$ & $856,0^{\mathrm{e}} \pm 34,0$ \\
\hline
\end{tabular}

Diferentes letras na mesma linha diferem significativamente pelo teste de Tukey $(\alpha=0,05)$.

Os resultados obtidos demonstraram que para o tempo de residência celular de 96 h, considerada a melhor condição experimental no desempenho térmico do forno, uma temperatura de chama de $1003,5^{\circ} \mathrm{C}$ foi atingida. Este tempo de residência corresponde ao período das máximas taxas de crescimento celular obtidas no fotobiorreator e que estão associadas às altas taxas de conversão de $\mathrm{CO}_{2}$, indicando que o crescimento microalgal está fortemente relacionado com a produção de $\mathrm{O}_{2}$, além de diversos COVs (Jacob-Lopes et al., 2010). Estes resultados podem ser melhor visualizados na Figura 2, que apresenta a curva de crescimento celular no fotobiorreator.

Figura 2 - Curva de crescimento celular da microalga Scenedesmus obliquus no fotobiorreator. 


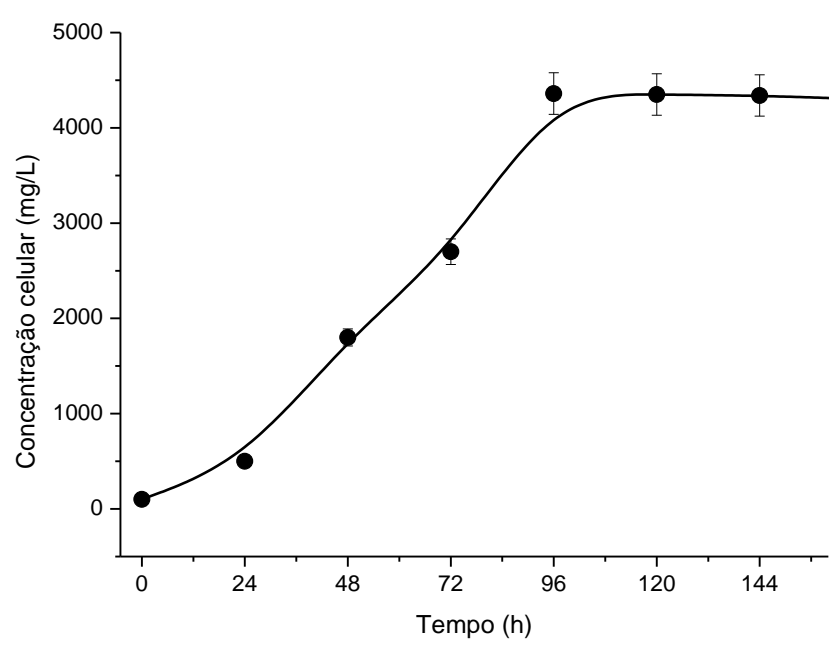

Para o ar atmosférico, a temperatura de chama foi de $907,3^{\circ} \mathrm{C}$, apresentando comportamento similar aos gases de exaustão do fotobiorreator. De acordo com $\mathrm{Bu}$ et al. (2016), esse fato ocorre devido ao fato de que o calor liberado e transferido durante a combustão é equivalente tanto em atmosferas contendo $\mathrm{O}_{2}$ e $\mathrm{N}_{2}$, quanto $\mathrm{O}_{2}$ e $\mathrm{CO}_{2}$.

Por outro lado, a corrente gasosa industrial simulada apresentou um comportamento mais diferenciado, com uma temperatura de chama de $822,8^{\circ} \mathrm{C}$, caracterizando um desempenho térmico insatisfatório. Baixas concentrações de oxigênio podem ocasionar a redução na temperatura de chama, resultando em uma menor eficiência térmica do sistema (Riahi et al., 2016). Além disso, as diferenças de temperatura estão relacionadas com as propriedades dos gases resultantes da oxicombustão $\left(\mathrm{CO}_{2}\right.$ e $\left.\mathrm{H}_{2} \mathrm{O}\right)$ e da combustão com ar $\left(\mathrm{N}_{2}\right)$, uma vez que moléculas triatômicas emitem maior energia por radiação do que moléculas diatômicas (Scheffknecht et al., 2011).

Dessa forma, os resultados obtidos demonstram a superioridade do uso dos gases de exaustão do fotobiorreator, os quais apresentam a potencialidade para serem utilizados como combustíveis gasosos em fornos de bio-oxicombustão.

\section{CONCLUSÃO}

Com base nos diferentes comburentes testados, obteve-se um aumento significativo na eficiência térmica do sistema com a injeção dos gases de exaustão do fotobiorreator, com uma temperatura de chama de $30,5 \%$ e $45,8 \%$ superiores ao uso do ar atmosférico e da corrente gasosa industrial simulada, respectivamente. A integração destes processos demonstrou ser uma potencial abordagem de engenharia para promover a sustentabilidade e economia dos processos industriais de combustão.

\section{REFERÊNCIAS}


APHA. Standard Methods for the Examination of Water and Wastewater, American Public Health Asssociation, 2005.

BU, C.; PALLARÈS, D.; CHEN X.; GÓMEZ-BAREA, A.; LIU, D.; LECKNER, B.; LU P. Oxy-fuel combustion of a single fuel particle in a fluidized bed: Char combustion characteristics, an experimental study. Chem. Eng. J., v. 287, p. 649-656, 2016.

CUÉLlAR-FRANCA, R. M.; AZAPAGIC, A. Carbon capture, storage and utilization technologies: A critical analysis and comparison of their life cycle environmental impacts. J. $\mathrm{CO}_{2}$ Utilization, v. 9, p. 82-102, 2015.

IPCC. Intergovernmental Panel on Climate Change. Climate Change 2014: Synthesis Report. IPCC, Geneva, Switzerland, 151 p., 2014.

JACOB-LOPES, E.; SCOPARO, C. H. G.; QUEIROZ, M. I.; FRANCO, T. T. Biotransformations of carbon dioxide in photobioreactors. Energy Conv. Manage., v. 51, p. 894-900, 2010.

JACOB-LOPES, E.; SEVERO, I. A.; BIZELLO, R. S.; TISCHER, B.; BARIN, J. S.; WAGNER, R.; MENEZES, C. R.; CICHOSKI, A. J.; ZEPKA, L.Q.; SUZUKI, S.; MENEZES, M. F. L.; PEREIRA, T. R.; ZOTTIN, L. S. Processo e sistema para reaproveitamento de gás carbônico transformados por meio de fotossíntese em oxigênio e hidrocarbonetos utilizados de forma integrada para aumento da eficiência térmica em sistemas de combustão. Patente n. PI 2015050276, 2015.

JIN, B.; SU, M.; ZHAO H.; ZHENG C. Plant wide control and operating strategy for air separation unit in oxy-combustion power plants. Energy Conv. Manage., v. 106, p. 782$792,2015$.

MARONEZE, M. M.; SIQUEIRA, S. F.; VENDRUSCOLO, R. G.; WAGNER, R.; MENEZES, C. R. de; ZEPKA L. Q.; JACOB-LOPES E. The role of photoperiods on photobioreactors - A potential strategy to reduce costs. Bioresour. Technol., v. 219, p. 493-499, 2016.

RIAHI, Z.; MERGHENI M. A.; SAUTET J. C.; NASRALLAH S. B. Experimental study of natural gas flame enriched by hydrogen and oxygen in a coaxial burner. Appl. Therm. Eng., v. 108, p. 287-295, 2016.

RIPKKA, R.; DERUELLES, J.; WATERBURY, J. B.; HERDMAN, M.; STANIER, R. Y. Generic Assignments, Strain Histories and Properties of Pure Cultures of Cyanobacteria. J. Gen. Microbiol., v. 111, p. 1-61, 1979.

SCHEFFKNECHT, G.; AL-MAKHADMEH, L.; SCHNELL, U.; MAIER J. Oxy-fuel combustion - A review of the state-of-the-art. In. J. Greenhouse Gas Control., v. 5, p. 16-35, 2011.

YIN, C.; YAN, J. Oxy-fuel combustion of pulverized fuels: Combustion fundamentals and modeling. Applied Energy, v. 162, p. 742-762, 2016. 